\title{
Interactive comment on "Data Assimilation of Argos profiles in North-west Pacific Model" by Z. Wang et al.
}

\section{Anonymous Referee \#2}

Received and published: 13 May 2016

General comments:

The aim of the paper is to show the development of an ocean operational system providing 5 days forecasts for the North-west Pacific using ROMS. Supposedly, a new method to determine the error-covariances for the Argo data is proposed, although it is not clearly shown in the text. The paper is poorly written, with grammatical errors that compromise its understanding and several points that need a deeper analysis and / or explanation. There is a lack of logic in the explanation of the experiments and the assimilation process, what makes it difficult to understand the reasoning for the methodology.

The assimilation process is poorly described. The "novel" background error covariance matrix determination method is not explained and the assimilated observations not

Printer-friendly version

Discussion paper 
presented.

The validation of the model results is not properly done. Other quantitative tools, such as Taylor diagrams are more appropriate for the task. Adequate datasets must be used for validation, instead of optimally interpolated maps. Monthly mean properties at a set level and one section do not provide a robust validation of the model results. Moreover the model is in part "validated" against the assimilated observations, which is not a valid approach. One should use independent observations and/or the analysis of important oceanographic process to perform a proper validation.

Therefore, I can not recommend this manuscript for publication in its present form.

Detailed comments:

Line 44: It is simplistic to say the forecast quality depends on the initial conditions. The surface forcing and boundary conditions are also determinant and usually updated in the assimilation process. Therefore, an assimilation system that focus only on the determination of optimal initial conditions is limited and may not perform well.

Line 57: You should provide some information that justifies your statement that the 4DVar scheme is to expensive for an operational system. It is in fact broadly used in operational forecast systems for diverse regions (eg: Powell et al. 2008 - Ocean Modelling). A comparison between the computational cost of the 4DVar and the proposed 3DVar schemes is necessary.

Line 75: What do you mean by "multivariate properties"?

Line 89: How did you smooth your bathymetry? Is the total volume of the basin conserved? How different is the smoothed bathymetry against the reality? Please keep in mind that strong changes in the slopes and modifications of the basin volume can have important effects on the dynamics.

Line 91: You say velocities (I assume both barotropic and baroclinic) and water levels are provided by SODA in the open boundaries. What about temperature and salinity?

Interactive

comment

Printer-friendly version

Discussion paper 
Line 106: In the "Assimilation algorithms" section you should explain how the errorcovariance and EOFs are calculated. Moreover, the use of EOFs for obtaining the vertical structure is one of many possible approaches and its use should not be generalized for 3DVar schemes (line 124). This whole section consists in a poor explanation of the 3DVar approach and do not touch the main point that is how you use it to assimilate Argo. It lack important information, such as: A description of your dataset; definition of your error-covariance matrix; it is not clear if you are using strong or week constrain;

Line 154: If your focus is on 5-days forecasts, how can the comparison of monthly mean SSTs be significant? Moreover, $150 \mathrm{~m}$ model temperatures are not representative of SST. Several datasets of SST, SLA, SSS and surface velocities are available for download for the validation of model results.

Line 175: At least difference maps for the AF and SF schemes as in Fig.3.1 should be generated to show some improvement of the assimilation results. Moreover, the use of optimal interpolation products is not recommended for the validation since it contains intrinsic decorrelation scales used in the interpolation. The validation should be done against real data!

Line 178: What does "very similar" means? You should avoid relative terms and show the actual numbers. A simple root-mean-squared deviation can give an idea of how close the model is to the observations.

Line 180: Why did you choose this section? You must justify it (Some process of particular interest?). The section must be shown in Fig. 2.1

Line 183-184: This phrase makes no sense...

Fig 3.3 and 3.4: Again, both AF and SF should be shown, together with difference plots.

Line 199 and Fig. 3.5: I assume by observations you mean Argo profiles. It is not a

Printer-friendly version

Discussion paper 
valid validation the comparison with the observations that were assimilated. It does not show any improvement of your model solution. In addition, it should be clearly stated NHESSD is these are mean profiles. If so, errorbars (standard deviation) must be added to the plots.

Section 3.2: This whole section is not valid, since independent observations must be used in the validation process.

Interactive

comment

Interactive comment on Nat. Hazards Earth Syst. Sci. Discuss., doi:10.5194/nhess-2016-53, 2016. 